\title{
Stickstoffmonoxyd in der Atemluft von Patienten mit interstitiellen Lungenkrankheiten
}

\author{
Nitric Oxide in Exhaled Breath of Patients with Interstitial Lung Diseases
}

Autor

Institut
J. Schildge

Medizin. Klinik Abt. Pneumologie, St. Vincentius-Kliniken Karlsruhe eingereicht 14.8.2010 akzeptiert nach Revision 27. 10.2010

\section{Bibliografie}

Dol http://dx.doi.org/ $10.1055 / \mathrm{s}-0030-1255958$

Online-Publikation: 29.11.2010

Pneumologie 2011; 65:

143-148 @ Georg Thieme

Verlag KG Stuttgart · New York ISSN 0934-8387

\section{Korrespondenzadresse}

Dr. med. Johannes Schildge

St. Vincentius - Kliniken

Karlsruhe gAG

Medizinische Klinik - Abteilung

Pneumologie

Südendstraße 32

76137 Karlsruhe

johannes.schildge@vincentiuska.de

\section{Zusammenfassung \\ $\nabla$}

Hintergrund: Die Konzentration von Stickstoffmonoxyd (NO) in der Atemluft findet als Entzündungsmarker bei Asthma bronchiale Verwendung. Ihre Rolle bei interstitiellen Lungenkrankheiten (ILK) ist bislang nicht geklärt.

Fragestellung: Die vorliegende Studie untersucht die NO-Konzentration in der Ausatemluft von Patienten mit ILK, wobei die NO-Konzentration mit konstantem Fluss und flussunabhängige NO-Parameter zur Bestimmung regionaler intrapulmonaler NO-Konzentrationen ermittelt werden. Diese Parameter werden mit klinischen, spirometrischen und bronchoskopischen Befunden der bronchoalveolären Lavage (BAL) verglichen. Methoden: 83 Patienten mit folgenden Diagnosen wurden in die Untersuchung eingeschlossen: 33 Patienten mit Sarkoidose, 14 Patienten mit idiopathischer Lungenfibrose, 12 Patienten mit Lungenbeteiligung im Rahmen einer Kollagenose, 10 Patienten mit exogen-allergischer Alveolitis, 8 Patienten mit kryptogener organisierender Pneumonie, 6 Patienten mit respiratorischer Bronchiolitis mit interstitieller Lungenkrankheit. 17 Patienten ohne nachweisbare ILK dienten als Kontrolle. NO wurde mit Hilfe eines ChemilumineszenzAnalysers bestimmt. Die NO-Konzentration in der Ausatemluft wurde bei konstantem Ausatemfluss von $50 \mathrm{ml} / \mathrm{s}$ entsprechend dem ATS/ERS-Standard (FENO50) und zusätzlich bei drei verschiedenen Flussraten von 30, 100 und $300 \mathrm{ml} / \mathrm{s}$ bestimmt, um die folgenden flussunabhängigen NO-Parameter zu errechnen: Die alveoläre NO-Konzentration (CANO), die NO-Konzentration in der Atemwegswand (CAWNO), die maximale NO-Flussrate in den Atemwegen (JAWNO) und die NO-Diffusionskapazität der Atemwege (DAWNO). Die BAL erfolgte im Rahmen einer flexiblen Bronchoskopie mit einem Spülvolumen von $100 \mathrm{ml}$ einer isotonen $\mathrm{NaCl}$-Lösung mit 5 Portionen von $20 \mathrm{ml}$.

\section{Abstract \\ $\nabla$}

Background: Nitric oxide (NO) in exhaled breath is a marker of inflammation in bronchial asthma. Its role in interstitial lung disease has so far not been established.

Objectives: The present study investigates exhaled NO in patients with interstitial lung diseases using constant flow and flow independent NO exchange parameters. These parameters were compared with clinical, lung function and BAL data.

Methods: 83 patients with the following diagnoses were included into the study: 33 patients with sarcoidosis, 14 patients with idiopathic pulmonary fibrosis, 12 patients with connective tissue disease affecting the lungs, 10 with extrinsic allergic alveolitis, 8 patients with cryptogenic organizing pneumonitis, 6 patients with respiratory bronchiolitis with interstitial lung disease and 17 control patients. NO was analysed using a chemiluminescence-analyser. NO-concentration in the exhaled breath was measured at a constant flow of $50 \mathrm{ml} / \mathrm{s}$ according the ATS/ERS-standard (FENO50) and additionally at three different flow rates of 30,100 and $300 \mathrm{ml} / \mathrm{s}$ to analyse the following flow independent NO parameters: NO alveolar concentration (CANO), airway wall NO concentration (CAWNO), maximum airway wall NO flux (JAWNO) and airway NO diffusing capacity (DAWNO). BAL was performed during flexible bronchoscopy with an irrigation volume of $100 \mathrm{ml}$ $0.9 \%$ saline solution in 5 aliquots of $20 \mathrm{ml}$ each.

Results: CANO $(p=0.012)$, CAWNO $(p=0.008)$ and DAWNO $(\mathrm{p}=0.031)$ varied between the diagnostic groups. CANO was positively correlated with age $(\mathrm{p}<0.05)$ and negatively correlated with inspiratory vital capacity (\%pred.; $p<0.01$ ). CAWNO was positively correlated with the relative proportion of CD8 cells in BAL $(p<0.01)$ and negatively correlated with smoking history $(\mathrm{p}<0.05)$. 
Ergebnisse: CANO ( $\mathrm{p}=0,012)$, CAWNO $(\mathrm{p}=0,008)$ und DAWNO $(\mathrm{p}=0,031)$ wurden von der Diagnose beeinflusst. CANO korrelierte positiv mit dem Patientenalter $(\mathrm{p}<0,05)$; negative Korrelationen bestanden mit der inspiratorischen Vitalkapazität (\%Soll; $\mathrm{p}<0,01)$. CAWNO korrelierte positiv mit dem Anteil der CD8-Zellen in der BAL $(p<0,01)$ und negativ mit der Nikotinanamnese in Packyears $(\mathrm{p}<0,05)$. DAWNO korrelierte negativ mit dem Anteil der CD8- Zellen in der BAL ( $p<0,05)$. FENO50 korrelierte positiv mit dem Alter $(\mathrm{p}<0,05)$ und negativ mit der Nikotinanamnese $(\mathrm{p}<0,01)$.

Schlussfolgerung: Bei Patienten mit interstitiellen Lungenkrankheiten fanden sich Zusammenhänge zwischen flussunabhängigen NO-Parametern und der Diagnose, spirometrischen und BAL-Variablen.
DAWNO was negatively correlated with the relative proportion of CD8 cells in BAL $(\mathrm{p}<0.05)$. FENO50 was positively correlated with age $(\mathrm{p}<0.01)$ and negatively correlated with smoking history $(\mathrm{p}<0.05)$.

Conclusions: In patients with interstitial lung diseases there were correlations of flow independent NO exchange parameters with diagnoses, lung function parameters and BAL-variables.

\section{Hintergrund}

Stickstoffmonoxyd (NO) wurde vor 20 Jahren erstmals in der Ausatemluft des Menschen nachgewiesen [1]. Weitere Studien zeigten eine vermehrte Konzentration von NO in der Ausatemluft bei allergischen Erkrankungen der Atemwege. Heutzutage wird NO in großem Umfang als nichtinvasiver Marker bei allergischem Asthma bronchiale eingesetzt [2-5]. Die American Thoracic Society (ATS) und die European Respiratory Society (ERS) formulierten Standards über die Technik und den Ablauf der NO-Messung in der Ausatemluft [6].

Bei nichtasthmatischen entzündlichen Lungenerkrankungen, insbesondere bei interstitiellen Lungenerkrankungen (ILK), ist die Rolle des NO als diagnostisches Werkzeug weniger gesichert. Bei granulomatösen Lungenerkrankungen wurde eine Zunahme der induzierbaren NO-Synthase (iNOS) im Lungengewebe nachgewiesen [7]. Manche Untersuchungen fanden eine erhöhte NOKonzentration in der Ausatemluft bei fibrosierenden Alveolitiden und Sarkoidose $[8,9]$.

Im Gegensatz zu anderen Gasen wie etwa Stickstoff oder Kohlendioxyd zeigt sich bei NO eine deutliche negative Korrelation zwischen Ausatemfluss und Konzentration in der Ausatemluft $[10,11]$. Diese Beziehung lässt sich am besten dadurch erklären, dass NO aus zwei Kompartimenten der Lunge stammt - den Atemwegen und der alveolären Region. Durch NO-Analyse bei verschiedenen Flussraten entwickelten einige Autoren mathematische Modelle, um flussunabhängige NO-Parameter bestimmen zu können. Der wesentliche Vorteil dieser Parameter liegt in der Möglichkeit, die lokalen NO-Konzentrationen in verschiedenen Lungenkompartimenten zu analysieren, den Atemwegen und dem Alveolarraum [12-14]. Eine wichtige Erkenntnis des Zweikompartimentmodells war die Information, dass die NO-Konzentration im Alveolarraum viel geringer ist als in den Atemwegen. Bei gesunden Erwachsenen wurden Konzentrationen von 50-250 ppb in den Atemwegen und von 0-4 ppb in den Alveolen ermittelt [15]. Deshalb ergibt die NO-Messung bei einem konstanten Fluss von $50 \mathrm{ml} / \mathrm{s}$ entsprechend den ATS/ERS-Standards möglicherweise keinen Einblick in den alveolaren Bereich, die bevorzugt betroffene Region bei ILK. Einige Studien, die Patienten mit exogen-allergischer Alveolitis, idiopathischer Lungenfibrose und Kollagenose mit Lungenbeteiligung untersuchten, fanden in der Tat erhöhte alveoläre NO-Konzentrationen [16-18].

Die bronchoalveoläre Lavage (BAL) ist als diagnostisches Verfahren bei entzündlichen Prozessen im alveolären Bereich weit verbreitet. Untersuchungen, welche bei Patienten mit ILK Resultate der erweiterten NO-Analytik mit BAL-Parametern vergleichen, wurden bislang nicht publiziert.

Die vorliegende Arbeit untersucht die diagnostische Wertigkeit der NO-Analyse in der Ausatemluft von Patienten mit ILK. Dabei wurden neben der ATS/ERS-Standard-Technik mit konstantem Fluss zusätzlich flussunabhängige NO-Parameter zur Ermittlung regionaler intrapulmonaler NO-Konzentrationen bestimmt. Die entsprechenden Resultate wurden mit klinischen, spirometrischen und BAL-Parametern verglichen.

\section{Methoden}

\section{$\nabla$}

Patienten ( $\bullet$ Tab. 1)

83 fortlaufende Patienten mit ILK wurden ausgewertet. Bei $46 \mathrm{~Pa}-$ tienten lag eine definitive histologische Diagnose vor, bei $37 \mathrm{~Pa}-$ tienten wurde die Diagnose aufgrund klinischer und radiologischer Befunde gestellt. Spirometrische Daten (Fluss-VolumenKurve) und die Nikotinanamnese wurden bei allen Patienten erhoben. 17 Patienten, bei denen eine Computertomographie eine interstitielle Lungenerkrankung nicht ausschließen konnte, Spirometrie, Bronchoskopie, BAL und transbronchiale Lungenbiopsie jedoch keine pathologischen Befunde zeigten, dienten als Kontrolle.

\section{NO-Analyse, flussunabhängige NO-Parameter}

NO wurde mit einem Chemiluminiszenz-Analyser mit integrierter Ultraschall-Spirometrie bestimmt; um eine Kontamination mit NO aus der Umgebungsluft zu vermeiden, inhalierten die Patienten NO-freie Luft (CLD88sp und Denox 88, Eco Medics, München, Deutschland). Das System erfüllt die Vorgaben der ATS/ERS und wurde vom Hersteller aufgerüstet, um NO bei unterschiedlichen Atemflussraten zu bestimmen.

Die flussunabhängigen NO-Parameter wurden nach der Technik und dem Rechenmodell von Högmann u. Mitarb. bestimmt [13]. Sie beschreiben die Alveolarregion über die Steady-State-Konzentration von NO in den Alveolen (Steady State Alveolar Concentration $=$ CANO $[\mathrm{ppb}]$ ), die Atemwegsregion über die NO-Konzentration in der Atemwegswand (Airway Wall NO Concentration $=$ CAWNO $[\mathrm{ppb}]$ ), die maximale NO-Flussrate aus der Atemwegswand (Maximum Total Volumetric Flux of NO from the Airway Wall = JAWNO $[\mathrm{pl} / \mathrm{s}]$ ) und die Diffusionskapazität für NO in den Atemwegen (Diffusing Capacity of NO in the Airways $=$ DAWNO $[\mathrm{ml} / \mathrm{s}]$ ). Hierzu wird die NO-Konzentration in der Ausatemluft bei drei verschiedenen Flussraten bestimmt: Bei geringem Fluss $(30 \mathrm{ml} / \mathrm{s})$, mittlerem Fluss $(100 \mathrm{ml} / \mathrm{s})$ und 
Tab. 1 Diagnosen, demografische Daten, Nikotinanamnese, spirometrische Parameter, NO-Parameter, BAL-Parameter (Mittelwerte \pm Standardabweichungen).

\begin{tabular}{|c|c|c|c|c|c|c|c|c|c|}
\hline & Sarkoidose & IPF & EAA & CTD & COP & RBILD & Kontrollen & p & Alle \\
\hline \multicolumn{10}{|l|}{ Patienten } \\
\hline n/weiblich & $33 / 8$ & $14 / 3$ & $10 / 7$ & $12 / 7$ & $8 / 5$ & $6 / 1$ & $17 / 8$ & $0,08 \pi$ & $100 / 39$ \\
\hline Alter, Jahre & $42,7 \pm 12,9$ & $68,4 \pm 6,9$ & $61,8 \pm 12,1$ & $63,0 \pm 9,3$ & $58,1 \pm 15,2$ & $57,0 \pm 12,6$ & $59,6 \pm 12,1$ & $<0,001$ & $55,6 \pm 15,0$ \\
\hline Pack-years & $11,8 \pm 16,9$ & $16,9 \pm 21,8$ & 0 & $16,4 \pm 16,6$ & $12,1 \pm 13,5$ & $46,0 \pm 5,5$ & $7,6 \pm 16,4$ & $<0,001$ & $12,5 \pm 18,4$ \\
\hline IVC, \%Soll & $89,6 \pm 17,1$ & $70,0 \pm 19,1$ & $88,7 \pm 15,8$ & $75,8 \pm 24,3$ & $86,6 \pm 23,1$ & $89,8 \pm 19,5$ & $102,4 \pm 15,8$ & $<0,001$ & $87,1 \pm 20,8$ \\
\hline FEV1, \%Soll & $88,8 \pm 21,3$ & $79,7 \pm 14,2$ & $94,4 \pm 17,7$ & $78,3 \pm 18,5$ & $79,0 \pm 22,7$ & $90,5 \pm 15,6$ & $101,6 \pm 17,1$ & 0,008 & $88,3 \pm 20,0$ \\
\hline FENO50, ppb & $19,8 \pm 11,5$ & $27,6 \pm 16,3$ & $24,7 \pm 8,73$ & $19,3 \pm 17,1$ & $19,5 \pm 6,09$ & $14,9 \pm 6,08$ & $23,6 \pm 10,1$ & 0,218 & $22,9 \pm 12,4$ \\
\hline CANO, ppb & $2,79 \pm 2,70$ & $4,37 \pm 3,98$ & $4,23 \pm 2,01$ & $6,21 \pm 4,44$ & $4,98 \pm 3,73$ & $2,52 \pm 1,90$ & $2,05 \pm 2,03$ & 0,012 & $3,52 \pm 3,10$ \\
\hline CAWNO, ppb & $74,7 \pm 183,0$ & $951 \pm 3086$ & $114 \pm 90,6$ & $839 \pm 1342$ & $3351 \pm 9345$ & $25,3 \pm 17,8$ & $71,7 \pm 71,0$ & 0,008 & $551 \pm 2908$ \\
\hline JAWNO, pl/sec & $1173 \pm 708$ & $1463 \pm 898$ & $1279 \pm 567$ & $1492 \pm 1347$ & $968 \pm 402$ & $1021 \pm 510$ & $1440 \pm 623$ & 0,614 & $1282 \pm 786$ \\
\hline DAWNO, $\mathrm{ml} / \mathrm{sec}$ & $36,7 \pm 24,7$ & $28,5 \pm 23,2$ & $24,4 \pm 26,3$ & $20,8 \pm 21,3$ & $21,6 \pm 10,7$ & $56,3 \pm 23,1$ & $23,1 \pm 17,5$ & 0,031 & $31,6 \pm 23,3$ \\
\hline \multicolumn{10}{|l|}{ BAL } \\
\hline Zellen, $\times 10^{6}$ & $15,1 \pm 11,1$ & $16,1 \pm 17,4$ & $27,0 \pm 18,5$ & $11,6 \pm 5,24$ & $12,0 \pm 9,30$ & $13,5 \pm 8,00$ & $5,71 \pm 1,91$ & $<0,001$ & $14,1 \pm 12,4$ \\
\hline Makrophagen, \% & $64,6 \pm 21,8$ & $69,1 \pm 26,4$ & $35,4 \pm 23,0$ & $64,6 \pm 19,6$ & $50,3 \pm 32,9$ & $88,1 \pm 13,6$ & $81,3 \pm 28,9$ & $<0,001$ & $65,4 \pm 27,3$ \\
\hline Lymphozyten, \% & $32,7 \pm 21,2$ & $8,78 \pm 7,46$ & $54,1 \pm 21,7$ & $23,6 \pm 17,1$ & $35,0 \pm 30,2$ & $6,67 \pm 6,02$ & $6,65 \pm 7,73$ & $<0,001$ & $24,9 \pm 23,1$ \\
\hline $\mathrm{N}(\mathrm{CD})$ & 23 & 3 & 10 & 6 & 6 & 0 & 0 & & 48 \\
\hline CD4\% & $73,7 \pm 15,6$ & $54,0 \pm 15,7$ & $67,7 \pm 26,5$ & $50,5 \pm 23,5$ & $75,5 \pm 13,7$ & n.u. & n.u. & 0,026 & $64,2 \pm 20,9$ \\
\hline CD8 \% & $20,6 \pm 14,2$ & $40,3 \pm 15,0$ & $33,7 \pm 30,9$ & $40,5 \pm 24,2$ & $19,2 \pm 12,5$ & n.u. & n. u. & 0,037 & $30,1 \pm 20,5$ \\
\hline $\mathrm{CD} 4 / \mathrm{CD} 8$ & $6,15 \pm 4,90$ & $1,58 \pm 0,93$ & $4,28 \pm 3,19$ & $1,87 \pm 1,47$ & $6,00 \pm 4,48$ & n.u. & n.u. & 0,023 & $4,27 \pm 4,25$ \\
\hline Neutrophile, \% & $1,63 \pm 1,76$ & $17,8 \pm 27,4$ & $4,10 \pm 4,30$ & $7,93 \pm 10,0$ & $10,4 \pm 11,3$ & $4,03 \pm 6,29$ & $1,74 \pm 2,42$ & $<0,001$ & $5,76 \pm 12,50$ \\
\hline Eosinophile, \% & $0,72 \pm 1,41$ & $3,46 \pm 2,03$ & $3,71 \pm 6,32$ & $3,46 \pm 8,04$ & $2,31 \pm 2,21$ & $0,83 \pm 1,57$ & $0,21 \pm 0,53$ & $<0,001$ & $1,78 \pm 3,81$ \\
\hline Mastzellen, \% & $0,21 \pm 0,33$ & $0,33 \pm 0,49$ & $0,75 \pm 1,30$ & $0,13 \pm 0,23$ & $0,59 \pm 0,74$ & $0,37 \pm 0,80$ & $0,06 \pm 0,12$ & 0,046 & $0,29 \pm 0,59$ \\
\hline Protein, mg/l & $235 \pm 184$ & $141 \pm 59,8$ & $552 \pm 527$ & $165 \pm 131$ & $271 \pm 237$ & $115 \pm 85,6$ & $98,7 \pm 39,5$ & 0,002 & $218 \pm 243$ \\
\hline
\end{tabular}

IPF = idiopathische Lungenfibrose; $E A A=$ exogen-allergische Alveolitis; $C T D=$ Kollagenose mit Lungenbefall; $C O P=$ kryptogene organisierende Pneumonie;

RBILD = respiratorische Bronchiolitis mit interstitieller Lungenerkrankung; FENO50 = NO-Konzentration in der Ausatemluft bei Atemstrom $50 \mathrm{ml} / \mathrm{sec} ; \mathrm{CANO}=\mathrm{Alveo}-$ läre NO-Konzentration; $C A W N O=$ NO-Konzentration in der Atemwegswand; AWNO = maximale NO-Flussrate aus der Atemwegswand; DAWNO = Diffusionskapazität

für NO in den Atemwegen; $\mathrm{N}(\mathrm{CD})$ = Anzahl der Patienten mit Lymphozytenmarkierung; $\mathrm{p}$ = Vergleich zwischen den Diagnosegruppen (Kruskal-Wallis-Test)

II $=X^{2}-$ Test; n. u. = nicht untersucht.

hohem Fluss $(300 \mathrm{ml} / \mathrm{s})$. Der Atemwegsdruck in der Ausatmung wird zwischen 5 und $20 \mathrm{~cm} \mathrm{H} 20$ gehalten, um NO-Beimischungen aus der Nasenhöhle zu vermeiden. Bei jeder Flussrate wird der Mittelwert aus drei Messungen mit jeweils einem Plateau über drei Sekunden bestimmt. CANO wird über eine Regressionsgerade aus den mittleren und hohen Flussraten bestimmt. CAWNO, DAWNO und JAWNO wurden aus allen drei Flussraten über eine iterative Technik errechnet.

Zusätzlich wurde die Konzentration von NO in der Ausatemluft entsprechend dem ATS/ERS-Standard bei einem konstanten Fluss von $50 \mathrm{ml} / \mathrm{s}$ gemessen (FENO50) [6].

Bei sämtlichen Patienten erfolgten die Bestimmung der spirometrischen Parameter und die NO-Analytik vor der Bronchoskopie.

\section{Bronchoskopie, BAL}

Die Bronchoskopie wurde am liegenden Patienten unter Lokalanästhesie mit 2\%iger Xylocainlösung mit einem flexiblen Videobronchoskop durchgeführt. Vor der Bronchoskopie erfolgte eine Inhalation von $2 \mathrm{ml}$ der Xylocainlösung über einen Düsenvernebler (Pari-Boy, Starnberg, Deutschland). Nach intravenöser Prämedikation mit 5-7,5 mg Midazolam wurde das Bronchoskop transoral eingeführt. Maximal $16 \mathrm{ml}$ der Xylocainlösung wurden durch den Instrumentierkanal des Bronchoskopes instilliert. Während der Untersuchung wurde angefeuchteter Sauerstoff mit einer Flussrate von 2 1/min über eine Nasensonde zugeführt. Die Sauerstoffsättigung wurde mit einem Pulsoxymeter über einen Fingersensor überwacht.

Bei generalisierten Lungenveränderungen wurde die BAL im Mittellappen und bei lokalisierten Veränderungen im bevorzugt betroffenen Lungensegment durchgeführt. Das Bronchoskop wurde in einem subsegmentalen Bronchus in Wedge-Position gebracht. 100 Milliliter sterile, isotone und auf $37^{\circ}$ gewärmte $\mathrm{NaCl}$-Lösung wurden in Portionen von $20 \mathrm{ml}$ über Einmalspritzen aus Plastik und einen im Instrumentierkanal liegenden Kunststoffkatheter instilliert und sofort reaspiriert. Alle Portionen wurden ausgewertet. Ein Rückgewinn von mindestens $30 \mathrm{ml}$ war Voraussetzung für Aufnahme in die Auswertung. Zwei transbronchiale Lungenbiopsien aus einem nicht lavagierten Segment wurden bei jedem Patienten entnommen.

\section{BAL-Analyse}

Das Volumen der BAL wurde gemessen und $5 \mathrm{ml}$ zur mikrobiologischen Analyse entnommen. Kompakte Partikel wurden durch Filtration über Gaze (Topper 8, Johnson \& Johnson, Norderstedt, Deutschland) entfernt, die BAL wurde in einem sterilen Plastikgefäß gesammelt. Die Gesamtzellzahl wurde durchflusszytometrisch (K800, Sysmex, Norderstedt, Deutschland) bestimmt. Zellpräparate wurden mit einer Zytozentrifuge angefertigt (Cytospin 2, Shandon, Frankfurt/Main, Deutschland). Die prozentuale Verteilung der Zellgruppen wurde durch mikroskopische Auszählung von 500 Zellen aus einem nach May-Grünwald-Giemsa gefärbten Zellausstrich ermittelt. 48 Patienten wiesen einen Lymphozytenanteil von über $15 \%$ auf. Bei diesen Patienten wurde die prozentuale Verteilung der Lymphozytenoberflächenmarker CD4 (T-Helferzellen) und CD8 (T-Suppressorzellen) bestimmt und der CD4/CD8-Quotient errechnet [19]. Zum Nachweis diente die APAAP-Methode (Immunkomplexe aus alkalischer Phosphatase und Antikörpern gegen alkalische Phosphatase).

Die Protein-Konzentration in der BAL wurde photometrisch bestimmt (Olympus AU 400, Hamburg, Deutschland). 
Tab. 2 Korrelationsanalyse zwischen NO-Parametern und Patienteneigenschaften (nichtparametrische Korrelationen nach Spearman). Angegeben sind die Korrelationskoeffizienten, in Klammer der p-Wert. Markierte Variablen haben in der logistischen Regression einen unabhängigen Einfluss.

\begin{tabular}{|c|c|c|c|c|c|}
\hline & FENO 50 & CANO & CAWNO & DAWNO & JAWNO \\
\hline Alter & $0,28(0,005)^{* *}$ & $0,31(0,006)^{*}$ & $0,32(0,001)$ & $-0,24(0,02)$ & $0,12(0,25)$ \\
\hline Pack years & $-0,31(0,002)^{*}$ & $-0,02(0,98)$ & $-0,33(0,001)^{*}$ & $0,23(0,02)$ & $-0,26(0,01)$ \\
\hline IVC \%Soll & $-0,14(0,18)$ & $-0,36(<0,001)^{* *}$ & $-0,17(0,10)$ & $0,07(0,49)$ & $-0,03(0,73)$ \\
\hline FEV1 \%Soll & $-0,05(0,63)$ & $-0,31(0,002)$ & $-0,07(0,49)$ & $0,02(0,82)$ & $0,02(0,86)$ \\
\hline FEV1/IVC. \% & $-0,06(0,59)$ & $0,21(0,04)^{*}$ & $0,12(0,23)$ & $-0,08(0,45)$ & $-0,05(0,57)$ \\
\hline Zellen in BAL & $-0,01(0,92)$ & $0,22(0,03)$ & $0,11(0,28)$ & $-0,17(0,09)$ & $-0,06(0,55)$ \\
\hline Makrophagen & $-0,12(0,22)$ & $-0,24(0,02)$ & $-0,07(0,51)$ & $0,05(0,59)$ & $-0,08(0,43)$ \\
\hline Neutrophile & $0,11(0,28)$ & $0,13(0,20)$ & $0,05(0,63)$ & $0,04(0,72)$ & $0,11(0,26)$ \\
\hline Eosinophile & $0,03(0,74)$ & $0,39(0,001)$ & $0,09(0,63)$ & $-0,09(0,39)$ & $-0,04(0,71)$ \\
\hline Mastzellen & $0,10(0,34)$ & $0,27(0,01)$ & $0,08(0,42)$ & $-0,12(0,23)$ & $0,01(0,93)$ \\
\hline Lymphozyten & $0,13(0,20)$ & $0,24(0,02)$ & $0,07(0,49)$ & $-0,10(0,31)$ & $-0,06(0,55)$ \\
\hline CD4 & $-0,20(0,16)$ & $-0,35(0,02)$ & $-0,35(0,01)$ & $0,28(0,05)$ & $-0,02(0,91)$ \\
\hline CD8 & $0,16(0,28)$ & $0,35(0,01)$ & $0,37(0,01)^{* *}$ & $-0,33(0,02)^{*}$ & $-0,01(0,95)$ \\
\hline CD4/CD8 & $-0,19(0,20)$ & $-0,34(0,02)$ & $-0,37(0,01)$ & $0,32(0,03)$ & $-0,02(0,88)$ \\
\hline Protein & $0,08(0,43)$ & $0,17(0,10)$ & $0,06(0,56)$ & $-0,10(0,33)$ & $0,04(0,69)$ \\
\hline
\end{tabular}

${ }^{*}=p<0,05 ;{ }^{* *}=p<0,01 ;$ Abkürzungen s. Tab. 1.

\section{Statistik}

Die Messreihen wurden zunächst auf Normalverteilung untersucht (PP-Plots, Shapiro-Wilk-Test). Da einige Werte nicht normal verteilt waren, kamen nichtparametrische Tests zur Anwendung. Unterschiede zwischen den verschiedenen Diagnosen wurden mithilfe des Kruskal-Wallis-Tests untersucht. Mehrfachvergleiche wurden nach Dunnett C korrigiert. Korrelationen zwischen den Variablen wurden über die nichtparametrische Korrelation nach Spearman errechnet. Zur Erkennung wechselseitiger Beeinflussungen der verschiedenen Variablen diente die logistische Regression. Da diese auf zwei Freiheitsgrade beschränkt ist, erfolgte die Berechnung mit den Medianen der Zahlenreihen als Grenzwerte: CANO 2,5 ppb, CAWNO $54 \mathrm{ppb}$, DAWNO $23 \mathrm{ppb} \times \mathrm{pl} / \mathrm{s}$. Eine Irrtumswahrscheinlichkeit $<0,05$ wurde als signifikant angenommen.

\section{Ergebnisse}

Allgemeine Betrachtung der Patienten ( $\bullet$ Tab. 1)

Alter, Nikotinkonsum, Spirometrie und BAL-Parameter waren zwischen den Diagnosen verschieden. Bei den Mehrfachvergleichen fanden sich folgende Unterschiede zur Kontrollgruppe $(p<0,05)$ : Patienten mit Sarkoidose waren jünger, Patienten mit RBILD rauchten mehr, Patienten mit IPF hatten eine geringere IVC \%soll und FEV1\%soll.

Bei den BAL-Parametern fanden sich folgende Unterschiede zur Kontrollgruppe: Patienten mit Sarkoidose hatten einen höheren Gehalt an Protein; Patienten mit Sarkoidose, EAA und Kollagenose hatten einen höheren Anteil an Lymphozyten; Patienten mit IPF hatten einen höheren Anteil an neutrophilen und eosinophilen Granulozyten.

\section{Vergleich der NO-Parameter zwischen den Diagnosen} CANO, CAWNO und DAWNO waren zwischen den Diagnosen signifikant verschieden. Im Mehrfachvergleich hatten Patienten mit Kollagenose ein höheres CANO als Patienten der Kontrollgruppe $(\mathrm{p}<0,05)$.

FENO50 und JAWNO waren zwischen den Diagnosen nicht verschieden.

\section{Korrelationen zwischen NO und den Krankheits- parametern ( Tab. 2)}

FENO50 war positiv mit dem Alter und negativ mit dem Inhalationsrauchen korreliert.

CANO war positiv mit dem Alter und dem Tiffeneau-Index korreliert. Negative Korrelationen zeigten sich mit der IVC\%soll ( $\bullet$ Abb. 1) und der FEV1\%soll.

Bei den BAL-Parametern fanden sich positive Korrelationen mit der Zellzahl, dem Anteil der eosinophilen Granulozyten, der Mastzellen, der Lymphozyten und der CD8-Lymphozyten. Negative Korrelationen fanden sich mit dem Anteil der Makrophagen, CD4-Lymphozyten und dem CD4/CD8-Quotienten.

CAWNO zeigte eine positive Korrelation mit dem Alter und eine negative Korrelation mit dem Zigarettenrauchen. Bei den BALParametern zeigte sich eine positive Korrelation mit dem Anteil der CD8-Lymphozyten ( $\bullet$ Abb. 2) und negative Korrelationen mit dem Anteil der CD4-Lymphozyten und dem CD4/CD8-Quotienten.

DAWNO war negativ mit dem Alter und positiv mit dem Zigarettenrauchen korreliert. Bei den BAL-Parametern fanden sich positive Korrelationen mit dem Anteil der CD4-Lymphozyten und dem CD4/CD8-Quotienten, daneben eine negative Korrelation mit dem Anteil der CD8-Lymphozyten.

JAWNO war negativ mit dem Zigarettenrauchen korreliert.

\section{Logistische Regression}

Die logistische Regression zeigte unabhängige Einflüsse auf FENO50 durch das Alter und das Zigarettenrauchen. CANO wurde durch das Alter, die IVC\%soll und den Tiffeneau-Index beeinflusst. CAWNO wurde durch das Zigarettenrauchen und den Anteil der CD8-Lymphozyten in der BAL beeinflusst. DAWNO wurde durch den Anteil der CD8-Lymphozyten in der BAL beeinflusst.

\section{Diskussion}

Während die Bestimmung der NO-Konzentration in der Ausatemluft mit konstantem Fluss bei Asthma bronchiale durch viele wissenschaftliche Studien untersucht wurde und weit verbreitet ist, ist die Aussage der NO-Analyse bei ILK bislang nicht geklärt. Erhöhte NO-Konzentrationen in der Atemluft von Patienten mit Sarkoidose und Systemsklerose mit Lungenmitbeteiligung wurden beobachtet $[8,9]$; jedoch konnten bei Sarkoidose keine Kor- 


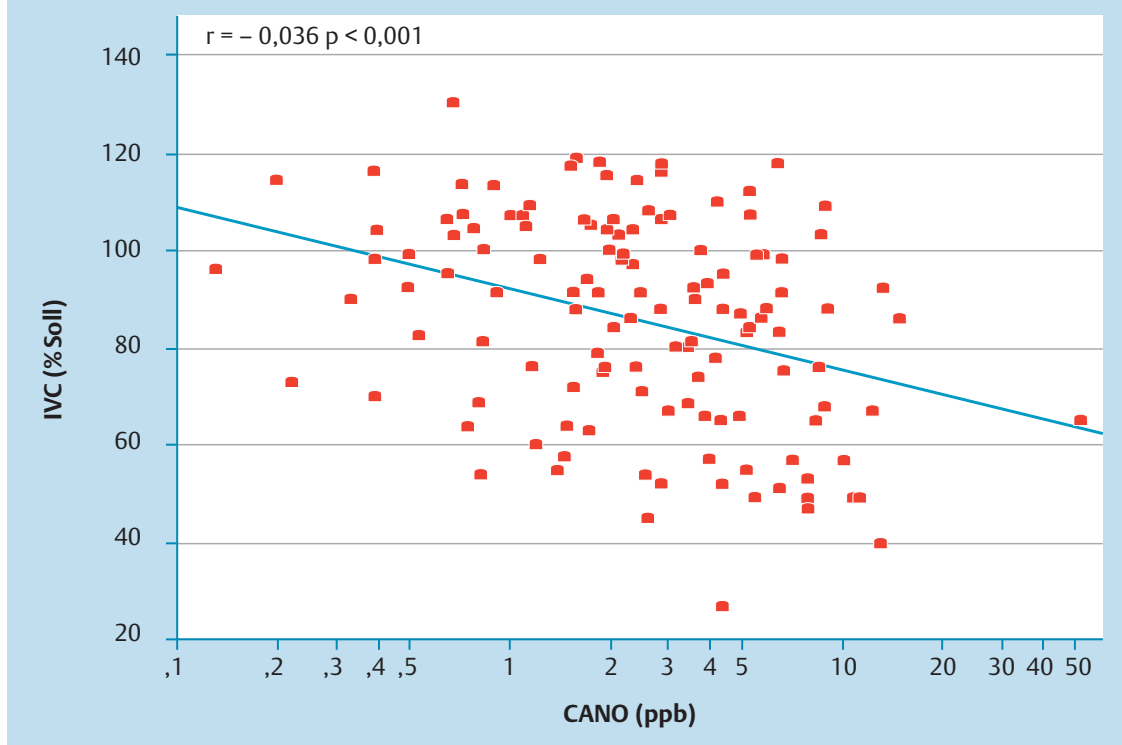

Abb. 1 Zusammenhang zwischen inspiratorischer Vitalkapazität und alveolärer NO-Konzentration (Scatter plot: IVC in \% Soll und log von CANO).

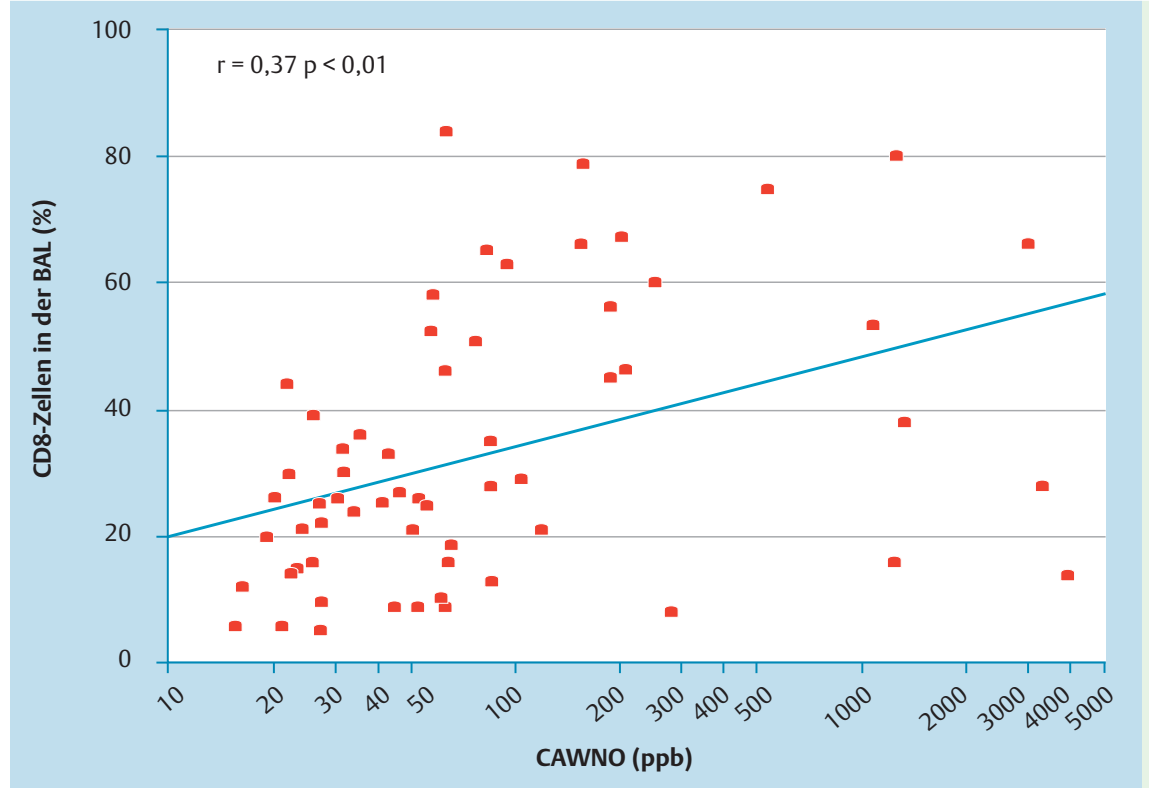

Abb. 2 Zusammenhang zwischen CD8-Lymphozyten in der BAL und der NO-Konzentration in der Atemwegswand (Scatter plot: CD8 und log von CAWNO).

relationen mit der Krankheitsaktivität nachgewiesen werden [9]. Meist wurden nur kleine Kollektive untersucht, Zusammenhänge zwischen der NO-Konzentration in der Atemluft und histologischen oder zytologischen Befunden wurden bislang nicht untersucht.

In der vorliegenden Arbeit wird die Höhe von FENO50 durch das Patientenalter und das Zigarettenrauchen beeinflusst: Mit zunehmendem Alter steigt FENO50, bei Inhalationsrauchern ist sie erniedrigt. Der niedrigere NO-Gehalt in der Atemluft von Rauchern ist gut bekannt. Offensichtlich führt Tabakrauch zu einer Suppression der iNOS. [20]. Die untersuchten Variablen einer ILK wie Diagnose, Lungenfunktion und BAL-Befunde hatten keinen Einfluss auf die Höhe von FENO50. Dies spricht dafür, dass in der untersuchten Serie die klinische und entzündliche Aktivität einer ILK die NO-Konzentration der Ausatemluft nicht nachweisbar beeinflusst.

Die flussunabhängige Analyse der NO-Konzentration in der Atemluft erlaubt die Bestimmung regionaler NO-Konzentrationen in verschiedenen Lungenkompartimenten.
Dabei stimmen die Messwerte der vorliegenden Kontrollgruppe mit kürzlich an einem großen Kollektiv Lungengesunder ermittelten Normwerten überein [15].

Es fanden sich Einflüsse der Diagnose auf die Höhe der Parameter CANO, CAWNO und DAWNO. CANO von Patienten mit Lungenbeteiligung im Rahmen einer Kollagenose war höher als bei Kontrollen. Ähnliche Resultate wurden von Tiev et al. mitgeteilt [17]. Die Korrelationsanalyse mit anschließender logistischer Regression zeigte mehrere voneinander unabhängige Korrelationen:

CANO korrelierte mit dem Alter und dem Ausmaß einer restriktiven Ventilationsstörung. Derartige Korrelationen mit dem Alter und der IVC wurden auch von Högman et al. [15] und Tiev et al. [17] mitgeteilt. Die positive Korrelation mit dem Ausmaß der Restriktion spricht dafür, dass CANO eine Rolle als Marker einer alveolären Schädigung spielen könnte. CAWNO war negativ mit dem Ausmaß des Inhalationsrauchens korreliert. Diesen Zusammenhang fanden auch Malinovschi et al. [20]. 
Der Einfluss der CD8-Lymphozyten auf den pulmonalen NOStoffwechsel im Sinne einer Zunahme von CAWNO und einer Abnahme von DAWNO, der in der vorliegenden Untersuchung nachweisbar war, wurde bislang nicht mitgeteilt. CD-8-Lymphozyten, auch zytotoxische T-Zellen genannt, stimulieren möglicherweise die iNOS.

Die referierten Ergebnisse sprechen dafür, dass es im Rahmen von ILK zu einer komplexen Beeinflussung des pulmonalen NOStoffwechsels kommt, wobei eine veränderte Ventilation und bronchoalveoläre Entzündungsphänomene eine Rolle spielen dürften. Zur Erfassung derartiger Vorgänge könnte die flussunabhängige NO-Analyse mit Bestimmung des regionalen pulmonalen NO-Stoffwechsels einen Beitrag leisten.

\section{Schlussfolgerung}

$\nabla$

Die vorliegende Untersuchung analysiert bei Patienten mit interstitiellen Lungenkrankheiten regionale intrapulmonale NO-Konzentrationen durch flussunabhängige NO-Bestimmung. Es zeigten sich Einflüsse durch die Diagnose, daneben Korrelationen mit dem Alter, dem Ausmaß des Inhalationsrauchens, mit spirometrischen und BAL-Parametern. Bei der standardisierten NOMessung mit konstantem Fluss fanden sich lediglich Korrelationen mit dem Inhalationsrauchen und dem Alter.

\section{Interessenkonflikt}

Der Autor gibt an, dass kein Interessenkonflikt besteht.

\section{Literatur}

1 Gustafsson LE, Leone AM, Person MG et al. Endogenous nitric oxide is present in the exhaled air of rabbits, guinea pigs and humans. Biochem Biophys Res Commun 1991; 181: 852-857

2 Alving K, Weitzberg E, Lundberg JM. Increased amount of nitric oxide in exhaled air of asthmatics. Eur Respir J 1993; 6: 1368 - 1370

3 Kharitonov SA, Yates D, Robbins RA et al. Increased nitric oxide in exhaled air of asthmatic patients. Lancet 1994; 343: 133-135

4 Massaro AF, Gaston B, Kita D et al. Expired nitric oxide levels during treatment of acute asthma. Am J Respir Crit Care Med 1995; 152: $800-803$
5 Yates DH, Kharitonov SA, Robbins RA et al. Effect of a nitric oxide synthase inhibitor and a glucocorticosteroid on exhaled nitric oxide. Am J Respir Crit Care Med 1995; 152: 892 - 896

6 ATS Board of Directors, and the ERS Executive Committee. ATS/ERS recommendation for standardized procedures for the online and offline measurement of exhaled lower respiratory nitric oxide and nasal nitric oxide, 2005. Am J Respir Crit Care Med 2005; 171: 912 - 930

7 Lakari E, Soini Y, Säily $M$ et al. Inducible nitric oxide synthase, but not xanthine oxidase, is highly expressed in interstitial pneumonias and granulomatous diseases of human lung. Am J Clin Pathol 2002; 117: $132-142$

8 Högman M, Stromberg S, Schedin U et al. Nitric oxide from the human respiratory tract efficiently quantified by standardised single breath measurements. Acta Physiol Scand 1997; 159: 345-346

9 Paredi P, Kharitonov SA, Loukides $S$ et al. Exhaled nitric oxide is increased in active fibrosing alveolitis. Chest 1999; 115: 1352 - 1356

10 Ziora D, Kauska K, Kozielski J. An increase in exhaled nitric oxide is not associated with activity in pulmonary sarcoidosis. Eur Respir J 2004; 24: 609-614

11 Silkoff PE, McClean PA, Slutsky AS et al. Marked flow-dependence of exhaled nitric oxide using a new technique to exclude nasal nitric oxide. Am J Respir Crit Care Med 1997; 155: 260-267

12 Tsoukias NM, George SC. A two-compartment model of pulmonary nitric oxide exchange dynamics. J Appl Physiol 1998; 85: 653-666

13 Högman M, Drca N, Ehrstedt C, Merilainen P. Exhaled nitric oxide partitioned into alveolar, lower airways and nasal contributions. Respir Med 2000; 94: 985 - 991

14 Silkoff PE, Serpil CE, Hunt JF. ATS workshop proceedings: Exhaled nitric oxide and nitric oxide oxidative metabolism in exhaled breath condensate. Proc Am Thorac Soc 2006; 3: 131 - 145

15 Högman M, Lafih J, Meriläinen P et al. Extended NO analysis in a healthy subgroup of a random sample from a Swedish population. Clin Physiol Funct Imaging 2009; 29: 18-23

16 Lehtimäki L, Kankaanranta H, Saarelainen S et al. Extended exhaled NO measurement differentiates between alveolar and bronchial inflammation. Am J Respir Crit Care Med 2001; 163: 1557-1561

17 Tiev KP, Cabane J, Aubourg F et al. Severity of scleroderma lung disease is related to alveolar concentration of nitric oxide. Eur Respir J 2007; 30: $26-30$

18 Tiev KP, Coste J, Ziani M et al. Diagnostic value of exhaled nitric oxide to detect interstitial lung disease in systemic sclerosis. Sarcoidosis Vasc Diffuse Lung Dis 2009; 26: $32-38$

19 Thomas L. Labor und Diagnose, ed 5. Frankfurt: TH Books, 1998: 1409 1415

20 Malinovschi A, Janson C, Holmkvist T et al. Effect of smoking on exhaled nitric oxide and flow-independent nitric oxide exchange parameters. Eur Respir J 2006; 28: 339-345 